\title{
Immunization of Guinea Pigs with Neisseria gonorrhoeae: Strain Specificity and Mechanisms of Immunity
}

\author{
By C. W. PENN, N. J. PARSONS, D. SEN, D. R. VEALE \\ AND H. SMITH \\ Department of Microbiology, University of Birmingham, Birmingham B $52 T T$
}

(Received I December I976)

SUMMARY

Infection of subcutaneously implanted chambers in guinea pigs conferred immunity against homologous infection of other chambers in the same animals. However, attempts to immunize guinea pigs by subcutaneous injection of filtered fluid from infected chambers, or with small doses of formalin-killed, chamber gonococci were not successful. Thus, neither organisms grown in vivo nor their extracellular products appeared to be exceptionally immunogenic.

In immunizing tests with different isolates of gonococci adapted to growth in guinea-pig chambers, cross-immunity to chamber infection with low challenge doses was detected only between two of six isolates. The killing of gonococci in chambers of immunized animals, which occurred only after homologous challenge or with the heterologous strain showing cross-immunity, was not due primarily to humoral factors in the chamber fluid but probably to an enhanced effectiveness of phagocytosis. The serum of immunized animals was bactericidal for homologous strains and for the strain showing cross-immunity but not for strains showing no cross-immunity. Hence, serum bactericidal activity might be a useful indicator for investigating the specificity of immunity produced by different gonococcal strains.

\section{INTRODUCTION}

Infection of perforated plastic chambers implanted subcutaneously into guinea pigs has been used to monitor immunity to gonococcal infection. This immunity was induced either by subcutaneous inoculations of large quantities of killed suspensions of gonococci (Arko, 1974; Turner \& Novotny, 1976) or by initial infection of chambers followed by spontaneous eradication of infection (Scales \& Kraus, 1974; Turner \& Novotny, 1976). Both methods prevented subsequent infection of chambers and elicited serum antibodies. When these antibodies were injected intramuscularly or introduced into the chambers 2 days before (Arko, 1974) or at the same time as (Scales \& Kraus, 1974) the challenge, they protected against infection. Immunity in the guinea-pig model, as in chimpanzees (Arko et al., 1974), appeared to be strain specific (Turner \& Novotny, 1976) but the large challenge doses used may have masked any weak cross-protection. Fresh serum from immunized animals killed gonococci in vitro but not after it was heated at $56{ }^{\circ} \mathrm{C}$ for $30 \mathrm{~min}$, indicating the possible involvement of complement (Scales \& Kraus, 1974). The antibacterial mechanisms within the chambers in vivo or the chamber fluid in vitro have not been investigated.

Gonococci adapted to growth in guinea-pig chambers formed smaller colonies on agar and were more resistant to killing by human serum and by human polymorphonuclear phagocytes than the parent strain grown in vitro. Washes of the organisms grown in vivo showed one or two precipitin lines in gel diffusion against antiserum produced by live infection; these were not shown by similar washes of organisms grown in vitro (Penn et al., 
I976; Witt et al., 1976). These differences between gonococci grown in vivo and in vitro suggested that the immunizing effect of chamber infection (Scales \& Kraus, r974; Turner \& Novotny, 1976), in which small numbers of gonococci $\left(\mathrm{I} \times 10^{6}\right.$ to $5 \times 10^{6} \mathrm{ml}^{-1}$; Veale et al., 1975) are present, may be due to a potent immunizing antigen not present to any significant extent in organisms grown in vitro. The latter are therefore needed in large quantities for producing effective immunity (Arko, 1974; Turner \& Novotny, 1976). Indeed, in a recent paper Arko, Bullard \& Duncan (1976) report that gonococci adapted to chambers in mice and grown once in vitro were more immunogenic for guinea pigs than the parent strain grown in vitro.

In the present study, gonococci (killed by formalin) and their products (filtered chamber fluid) have been collected from guinea-pig chambers (Penn et al., 1976) and their immunizing activities compared with those of the killed in vitro-grown parent strain. Strain specificity of immunity has been investigated using low challenge doses of chamber-adapted organisms. Finally, the antibacterial mechanisms within chambers were studied by comparing the multiplication or killing of gonococci in chambers in vivo, in chamber fluid (in vitro) and in serum (in vitro) of non-immune guinea pigs and of those immunized with strains homologous and heterologous to those used in the tests. The latter experiments suggested a possible indicator of immunity in guinea pigs for future studies on the specificity of immunizing activity amongst gonococcal strains.

\section{METHODS}

Gonococcal strains. The pilated, small-colony-forming strains As and BS, resembling Kellogg types $\mathrm{I}$ and 2 and infective for guinea-pig chambers, were described by Veale et al. (1975). Strain DS was another similar laboratory strain. Strains CS, ES and FS were smallcolony isolates obtained from urethral pus by two cultures on AG agar (Penn et al., 1976). Strain BS3, i.e. strain BS passaged three times through guinea-pig chambers and frozen in liquid nitrogen, was described by Penn et al. (I976). Strains AS3, CS3, DS3, ES3 and FS3 were derived similarly from strains AS, CS, DS, ES and FS respectively by passaging three times through the chambers. The approximate minimal infective doses (m.i.d.) for guinea-pig chambers were $<20$ organisms for $\mathrm{BS}_{3}, \mathrm{As}_{3}, \mathrm{Cs}_{3}, \mathrm{DS}_{3}, \mathrm{ES}_{3}$ and $\mathrm{FS} 3$.

Culture media. Media for growing gonococci, for diluting suspensions and for viable counts were described by Penn et al. (1976).

Subcutaneously implanted guinea-pig chambers. Male Dunkin-Hartley guinea pigs (grade 2; Olac, Banbury, Oxfordshire; about 600 g) received two chambers which were implanted, inoculated and sampled as described by Veale et al. (1975).

Killed gonococci and chamber fluid for immunization experiments. Strain BS was grown on AG agar (Penn et al., 1976) for $20 \mathrm{~h}$ and suspended $\left(5 \times 10^{6}\right.$ gonococci $\left.\mathrm{ml}^{-1}\right)$ in PBS (Penn et al., 1976). Strain BS3 (about 1000 organisms) was inoculated into chambers. Fluid (viable count $\mathrm{I} \times 1 \mathrm{I}^{6}$ to $5 \times 10^{6} \mathrm{ml}^{-1}$ ) was removed after $24 \mathrm{~h}$ and centrifuged at $200 \mathrm{~g}$ for $5 \mathrm{~min}$. The supernatant fluid was centrifuged at $3000 \mathrm{~g}$ for $\mathrm{I} 5 \mathrm{~min}$ and filtered through Millipore membranes $(0.22 \mu \mathrm{m}$ pore size). The pellet was suspended in PBS to the original volume of chamber fluid (i.e. $\mathrm{I} \times 10^{6}$ to $5 \times 10^{6}$ organisms $\mathrm{ml}^{-1}$ ). These chamber-grown organisms (strain BS4) and BS organisms were killed by treatment with $0.25 \%(\mathrm{v} / \mathrm{v})$ formalin at $4{ }^{\circ} \mathrm{C}$ overnight. Sterility was tested by plating $0.1 \mathrm{ml}$ of the treated suspension on haemoglobin agar. The killed gonococci and filtered chamber fluid were stored at $-20^{\circ} \mathrm{C}$ in $\mathrm{I} \mathrm{ml}$ portions.

Immunization of guinea pigs by infection of subcutaneous chambers. Guinea pigs implanted 
with two chambers were inoculated in one chamber with strain BS3 (IO0O organisms, about 500 m.i.d.). Four to six weeks later the opposite chamber was challenged with strain BS3 ( 1000 organisms, about 500 m.i.d.). Animals were regarded as immune if viable gonococci could not be detected in the chamber fluid $\left(<50 \mathrm{ml}^{-1}\right) 5$ days after challenge.

Experiments on cross-immunization between strains $\mathrm{BS}_{3}, \mathrm{AS}_{3}, \mathrm{CS}_{3}, \mathrm{DS}_{3}, \mathrm{ES}_{3}$ and $\mathrm{FS}_{3}$ were conducted similarly with about $500 \mathrm{~m}$.i.d. of organisms for both initial inoculation and subsequent challenge.

Immunization of guinea pigs with killed gonococci and their products. Guinea pigs were implanted with two chambers and I week later received (subcutaneously) killed strain BS organisms $\left(\mathrm{I} \times 1 \mathrm{IO}^{6}\right)$, killed strain $\mathrm{BS} 4$ organisms $\left(\mathrm{I} \times 1 \mathrm{O}^{6}\right)$ or filtered chamber fluid (a volume that contained either $\mathrm{I} \times 10^{6}$ or $4 \times 10^{6}$ strain BS4 organisms; about 0.2 or $\left.0.8 \mathrm{ml}\right)$. Control animals were implanted simultaneously but were not inoculated. The subcutaneous injections were repeated four times at weekly intervals. One week after the last injection $0.25 \mathrm{ml}$ of fluid was removed from each chamber for checks on sterility (haemoglobin agar; $24 \mathrm{~h}$ incubation). The chambers were then challenged with strain BS3 organisms (50 to 250 organisms; about 25 to I 25 m.i.d.) in TSB. Viable counts on samples of the chamber fluids 5 days after challenge showed whether or not the animals were immune $(<50$ gonococci per $\mathrm{ml}$ chamber fluid). Chamber fluid of challenged control animals contained $0.5 \times 10^{6}$ to $5^{\circ} \mathrm{O} \times \mathrm{IO}^{\mathbf{6}}$ gonococci $\mathrm{ml}^{-1}$.

Kinetics of growth and killing in subcutaneous chambers. Chambers were inoculated with $\mathrm{I} \times \mathrm{IO}^{5}$ gonococci ( $\mathrm{BS}_{3}, \mathrm{AS}_{3}$ or $\left.\mathrm{CS}_{3}\right)$ in TSB $(0.2 \mathrm{ml})$ after diluting frozen stocks immediately after thawing. Samples $(0.05 \mathrm{ml})$ of fluid were withdrawn $\mathrm{I}, 2,4$ and $7 \mathrm{~h}$ after challenge for viable counts.

Kinetics of growth and killing in guinea-pig chamber fluid or serum. Recently withdrawn chamber fluid $(0.5 \mathrm{ml})$ or freshly prepared serum $(0.5 \mathrm{ml})$ from normal guinea pigs or those immunized by chamber infection, was mixed with a suspension $(0.05 \mathrm{ml})$ of gonococci (about $5 \times \mathrm{IO}^{5}$ organisms $\mathrm{ml}^{-1}$ ) in TSB in a $2 \mathrm{ml}$ screw-capped polypropylene tube and incubated in $5 \% \mathrm{CO}_{2} / 95 \%$ air at $37{ }^{\circ} \mathrm{C}$. Samples $(0.05 \mathrm{ml})$ for viable counts were withdrawn after I, 2 and $4 \mathrm{~h}$ and occasionally after $3 \mathrm{~h}$.

Complement concentration in chamber fluid. Sheep red blood cells (SRBC) in Alsever's solution (Wellcome Reagents) were washed in 10 vols barbitone buffer $\mathrm{pH} 7 \cdot 2$ (Kabat \& Mayer, 196I) and suspended in the buffer to $4 \%(\mathrm{v} / \mathrm{v})$ (volume of SRBC measured after centrifuging at $500 \mathrm{~g}$ for $5 \mathrm{~min}$ ). The suspension was mixed with an equal volume of heated $\left(56^{\circ} \mathrm{C}, 30 \mathrm{~min}\right.$ ) rabbit anti-SRBC serum (Wellcome Reagents) and kept at $37^{\circ} \mathrm{C}$ for $2 \mathrm{~h}$. The suspension of sensitized cells was then added to equal volumes of twofold dilutions of chamber fluid in PBS. The complement titre was the dilution producing approximately $50 \%$ lysis. Controls included a standard preparation of freeze-dried guinea-pig complement (Wellcome Reagents) which was stored at $-70^{\circ} \mathrm{C}$.

\section{RES ULTS}

Attempts to immunize guinea pigs with killed gonococci grown in vivo (BS4) and in vitro (BS) and with chamber fluid

Infection of chambers with strain BS 3 in six guinea pigs protected the second chambers in each animal against challenge with BS 34 to 6 weeks later. Since the numbers of gonococci that could be harvested from the chambers were relatively small compared with the doses of organisms used in previous studies (Arko, 1974; Turner \& Novotny, 1976), the comparisons of immunizing activities of the strains grown in vivo and in vitro had to be done with 
Table I. Attempts to immunize guinea pigs with killed gonococci grown in vivo (BS4) and in vitro (BS) and with filtered chamber fluid

Inocula*

Experiment I :

Killed strain BS $\left(\mathrm{I} \times 10^{6}\right)$

Killed strain BS4 $\left(\mathrm{I} \times 10^{6}\right)$

Chamber fluid $\left(0.2 \mathrm{ml} \equiv \mathrm{I} \times 10^{6} \mathrm{BS} 4\right)$

Controls

Experiments 2 and 3 :

Chamber fluid $\left(0.8 \mathrm{ml} \equiv 4 \times 10^{6} \mathrm{BS} 4\right)$

Controls $\frac{\text { No. of chambers found immunet }}{\text { No. of chambers inoculated } \ddagger}$

$$
\begin{aligned}
& 0 / 7 \\
& 3 / 8 \\
& 1 / 10 \\
& 0 / 7 \\
& \\
& 2 / 16 \\
& 0 / 15
\end{aligned}
$$

\begin{tabular}{|c|c|c|c|c|c|c|c|}
\hline \multirow{2}{*}{$\begin{array}{l}\text { Immunizing } \\
\text { strain* }\end{array}$} & \multirow{2}{*}{$\begin{array}{l}\text { Challenging } \\
\text { strain* } \ldots\end{array}$} & \multicolumn{6}{|c|}{$\frac{\text { No. of chambers found immune }}{\text { No. of chambers inoculated }}$} \\
\hline & & BS3 & $\mathrm{AS3}$ & $\mathrm{Cs} 3$ & DS3 & ES3 & FS3 \\
\hline BS3 & & IO/IO & $5 / 5$ & $0 / 4$ & NT & NT & NT \\
\hline AS3 & & $3 / 3$ & $3 / 3$ & $0 / 3$ & NT & NT & NT \\
\hline Cs3 & & $0 / 3$ & $0 / 3$ & $3 / 3$ & NT & NT & NT \\
\hline DS3 & & $0 / 3$ & NT & $0 / 3$ & $4 / 5$ & NT & NT \\
\hline ES3 & & $0 / 3$ & NT & $0 / 3$ & NT & $6 / 6$ & NT \\
\hline FS3 & & $0 / 3$ & NT & $0 / 3$ & NT & NT & $6 / 6$ \\
\hline
\end{tabular}

* Five inocula were given at weekly intervals.

+ Less than 50 gonococci per $\mathrm{ml}$ chamber fluid after 5 days.

$\ddagger 25$ to 125 m.i.d. of strain BS3.

Table 2. Immunization of guinea pigs by infection with different strains gonococci, showing the results of homologous and heterologous challenges

NT, Not tested.

* Approximately 500 m.i.d. was used in one chamber for immunization and also for challenge of the second chamber on the same animal.

$\dagger$ Less than 50 gonococci per $\mathrm{ml}$ chamber fluid after 5 days.

low inocula. Therefore a low challenge dose (25 to 125 m.i.d. of strain BS3) was used in the hope of detecting low levels of immunity. However, neither the killed organisms grown in vivo nor the filtered chamber fluid contained a powerful immunizing antigen (Table I). Only in a few animals was immunity detected despite the low challenge. No animals acquired immunity from corresponding small doses of the strain grown in vitro (Table I) although in other experiments, doses Ioo-fold higher produced significant immunity.

\section{Strain specificity in cross-immunity experiments with gonococcal strains}

There was considerable strain specificity in the immunity produced in guinea pigs by chamber infection with six strains adapted to growth in guinea-pig chambers (Table 2). Immunity to challenge by strain BS3 was evoked only by itself and by strain As3; immunity to challenge by strain $\mathrm{CS}_{3}$ was raised only by itself. Strains DS3, ES3 and FS3 immunized against homologous challenge but not heterologous challenge with strains BS 3 and $\operatorname{cs}_{3}$. As expected, immunization with either $\mathrm{AS}_{3}$ or $\mathrm{BS}_{3}$ was effective against challenge with $\mathrm{AS}_{3}$. Thus crossimmunity was detected only between strains As 3 and Bs3 although there may also have been cross-immunity between strains DS3, ES3 and FS3. 


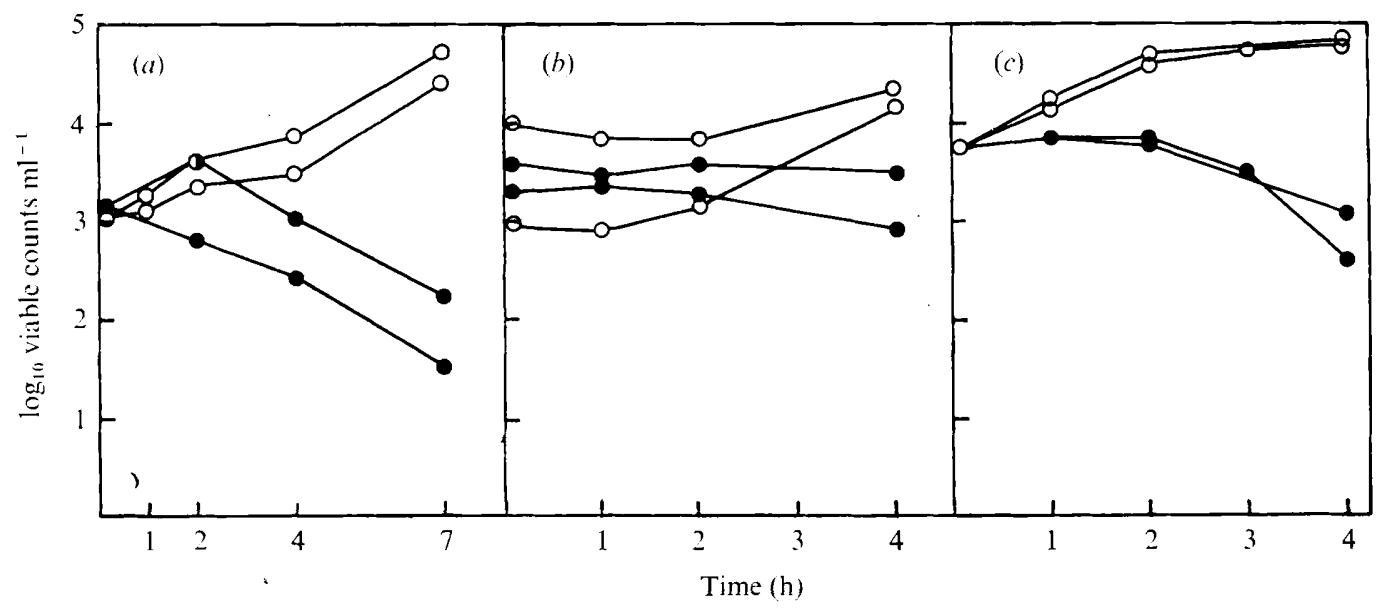

Fig. I. Growth and killing of strain BS3 in (a) guinea-pig chambers, $(b)$ fluid from guinea-pig chambers, and $(c)$ sera from guinea pigs: $(O)$ normal guinea pigs; $(O)$ guinea pigs immunized against strain Bs 3 by live chamber infection. The results are from two experiments which were typical of five or six conducted.

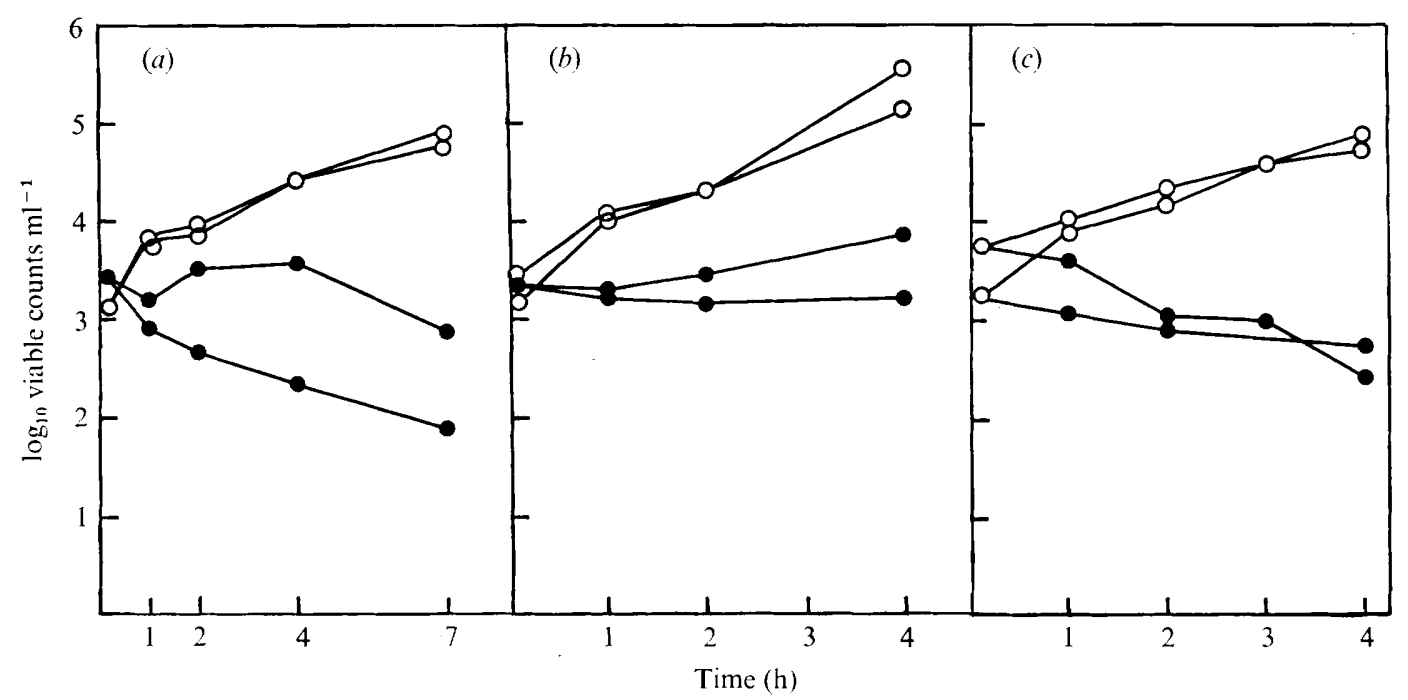

Fig. 2. Growth and killing of strain As3 in (a) guinea-pig chambers, (b) fluid from guinea-pig chambers, and $(c)$ sera from guinea pigs: $(O)$ normal guinea pigs; $(O)$ guinea pigs immunized against strain Bs3 by live chamber infection. The results are from two typical experiments.

The behaviour of strain $\mathrm{BS} 3$ in chambers in vivo, in chamber fluid and in serum of normal guinea pigs and of those immunized by chamber infection with $\mathrm{BS} 3$

In the chambers of non-immunized animals, strain Bs3 multiplied whereas in chambers of immunized animals the count declined in 4 to $7 \mathrm{~h}$ (Fig. $\mathrm{r} a$ ). In chamber fluid from normal animals, strain BS3 multiplied whereas in fluid from immunized animals essentially no changes in viable counts occurred (Fig. I $b$ ). In normal serum the counts of $\mathrm{BS}_{3}$ remained static but were reduced in sera from immunized animals (Fig. I $c$ ). 


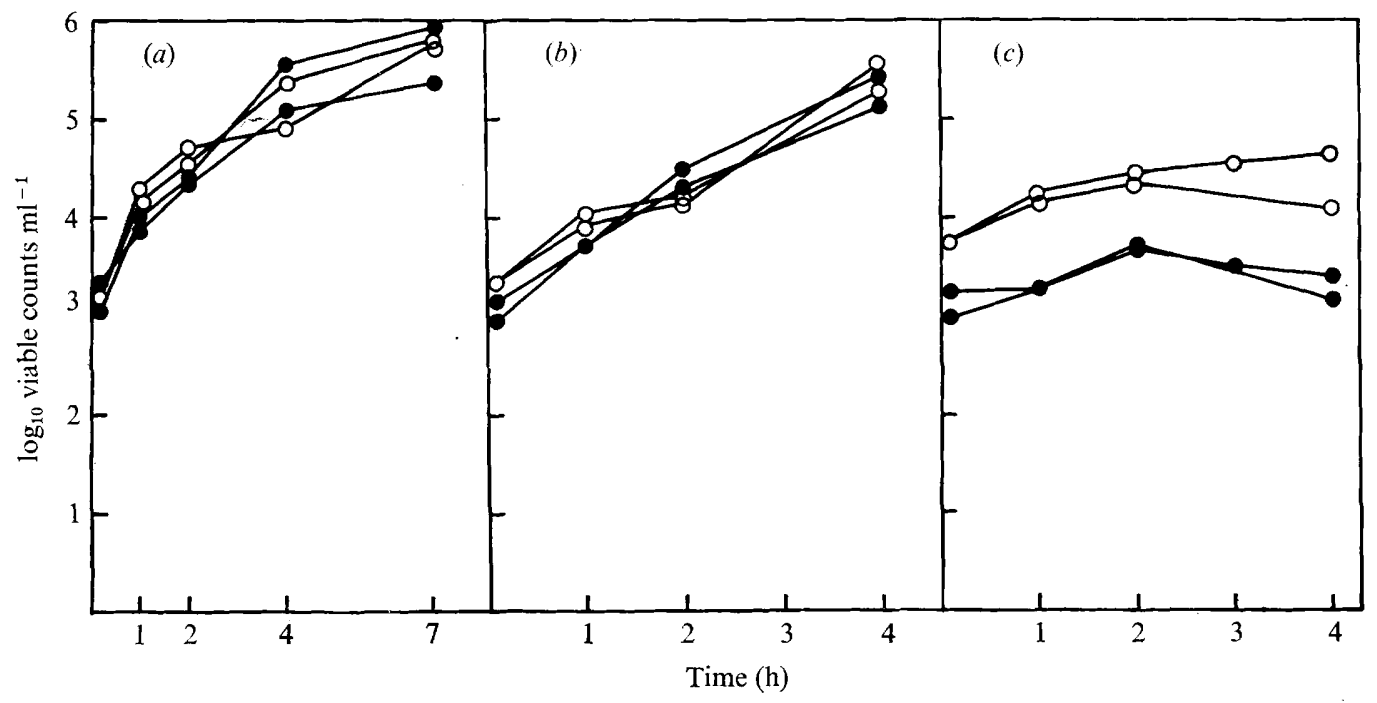

Fig. 3. Growth and killing of strain cs3 in (a) guinea-pig chambers, (b) fluid from guinea-pig chambers, and $(c)$ sera from guinea pigs: $(O)$ normal guinea pigs; $(\bullet)$ guinea pigs immunized against strain BS3 by live chamber infection. The results are from two typical experiments.

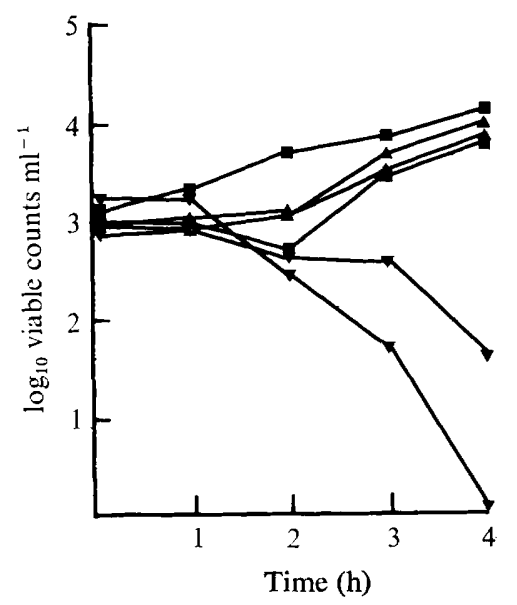

Fig. 4. Growth and killing of strains BS3 $(\boldsymbol{\nabla}), \operatorname{As} 3(\boldsymbol{\Delta})$ and $\operatorname{cs} 3(\boldsymbol{\nabla})$ in antiserum from guinea pigs immunized by chamber infection with strain cs3. The results are from two typical experiments.

In three animals complement titres in non-infected chamber fluid were much less ( $\mathrm{I}: 8$ to $\mathrm{I}: 16)$ than those in serum from the same animals ( $I: 64$ to $I: I 28)$. After infection, complement titres in fluid remained at about $\mathrm{I}: 8$ for $\mathrm{I}$ to 2 weeks, then fell to $\mathrm{I}: 2$, or less, at 4 to 6 weeks, by which time the chamber contents were purulent.

The behaviour of strains $\mathrm{AS}_{3}$ and $\mathrm{Cs}_{3}$ in chambers in vivo, in chamber fluid and in serum of normal guinea pigs and of those immunized by chamber infection with $\mathrm{BS} 3$

In kinetic experiments strain AS3, which showed cross-immunity with strain BS3 (Table 2), behaved in a manner similar to $\mathrm{BS}_{3}$ in chambers, in fluid and in serum of non-immune guinea pigs and of guinea pigs immunized by strain Bs3. Thus, in the chambers (Fig. 2a) 
As3 multiplied in non-immune animals and was reduced in immune animals. In chamber fluid (Fig. $2 b$ ) from non-immune animals multiplication occurred, whereas in that from immune animals viable counts showed little change. In the serum (Fig. $2 c$ ) from nonimmune animals there was a small increase in numbers of viable AS3 but numbers decreased in serum from immunized animals.

In contrast with the behaviour of AS3 and in accord with the lack of cross-immunity between $\mathrm{Cs}_{3}$ and $\mathrm{BS}_{3}$ (Table 2), the behaviour of $\mathrm{Cs}_{3}$ in chambers, in chamber fluid and in serum was similar for non-immune animals and those immunized with BS3 (Fig. 3). In particular there was no reduction of viable counts in the chambers and in the serum of immunized animals. The correlation between serum bactericidal activity and chamber immunity, suggested by the results of experiments with strains BS3, AS 3 and $\operatorname{Cs} 3$ in chambers and sera from animals immunized with strain $\mathrm{Bs} 3$, received support from the results summarized in Fig. 4: strain $\operatorname{Cs} 3$ was killed by antiserum from guinea pigs immunized by chamber infection with itself, but this antiserum had no effect on strains BS3 and AS3 with which cs 3 showed no cross-immunity (Table 2 ).

\section{DISCUSSION}

Although the immunizing effect of chamber infection has been confirmed, no evidence was obtained for the liberation of a potent extracellular antigen during such infection nor for the possession by chamber-grown gonococci of immunizing capacity greatly superior to that of organisms grown in vitro. Only small immunizing doses of killed gonococci could be used because of the limited supply of organisms grown in vivo. These doses were about Ioo-fold less than the numbers of killed organisms grown in vitro which were shown to be immunogenic by others (see Introduction) and ourselves. However, had the organisms grown in vivo possessed unusual immunizing potency, the negative results against a low challenge (Table I) might not have been obtained.

In tests with six different isolates of gonococci, cross-immunizing activity was detected only between two isolates (AS3 and BS3) although additional cross-immunity might have been detected had all combinations with strains DS3, ES3 and FS3 been tested (Table 2). In these tests the use of high challenge doses, which might break through any existing cross-immunity, was avoided. These results suggest that strain specificity may be a major difficulty in the design of a vaccine against gonorrhoea. The extent of the problem is difficult to gauge because sufficient experiments on the specificity of immunizing activity of different gonococcal serotypes (e.g. the I6 described by Johnston, Holmes \& Gotschlich, I976) have not yet been conducted.

In experiments with three isolates $\mathrm{BS}_{3}, \mathrm{AS}_{3}$ and $\mathrm{CS}_{3}$, two of which (AS3 and $\mathrm{BS}_{3}$ ) showed cross-immunity, the progress of chamber infection in animals immunized by $\mathrm{BS}_{3}$ was as expected. Counts of $\mathrm{BS}_{3}$ and $\mathrm{AS}_{3}$ decreased and those of $\mathrm{Cs}_{3}$ increased. However, the chamber fluid of the immunized animals had no marked bactericidal effect on $\mathrm{BS}_{3}$ and AS3 organisms although there was no increase in count as occurred in fluid from chambers of normal animals. Although some humoral bactericidal or bacteriostatic activity may have been present, the observed complement level, about one tenth of that found in serum, might not have been sufficient for optimum bactericidal activity. Probably the major effect of immunization was to generate opsonic antibody, thereby increasing the efficiency of the phagocytes which enter the chamber after infection. These results are in accord with previous observations on chambers and chamber fluid of non-immunized guinea pigs infected with the parent BS strain of gonococci (Veale et al., 1977). They indicated phagocytosis 
rather than humoral factors as the main defence mechanism of the chambers during establishment of infection.

As Scales \& Kraus (I974) also report, the serum of immunized animals was bactericidal. Furthermore, with the few strains tested, there was a correlation between this bactericidal activity and immunity to chamber infection. Thus, the serum from guinea pigs immunized with strain BS3 was bactericidal for the strain showing cross-immunity (AS3) but not for strain CS3 which showed no cross-immunity with strain BS3. Also, the serum of animals immunized with strain $\operatorname{cs} 3$ was bactericidal only for the homologous strain and not for two strains (AS3 and BS3) with which it showed no cross-immunity. Serum bactericidal activity may therefore be a useful indicator of immunity to gonococci, at least in the guinea pig; and, being assessed by a quick and convenient test, may be useful for gauging the extent of strain specificity in immunity to gonorrhoea. This would be necessary for assessing the potential of a gonococcal vaccine.

C. W. Penn is a Beit Memorial Fellow.

\section{REFERENCES}

ARKo, R. J. (1974). An immunologic model in laboratory animals for the study of Neisseria gonorrhoeae. Journal of Infectious Diseases 129, 45I-455.

ARko, R. J., Bullard, J. C. \& DunCAN, W. P. (1976). Effects of laboratory maintenance on the nature of surface active antigens of Neisseria gonorrhoeae. British Journal of Venereal Disease 52, 316-325.

Arko, R. J., Kraus, S. J., Brown, W. J., Buchanan, T. M. \& KUhN, U. S. G. (1974). Neisseria gonorrhoeae: effect of systemic immunization on resistance of chimpanzees to urethral infection. Journal of Infectious Diseases 130, $160-164$.

KABAT, E. A. \& MAYER, M. M. (1961). Experimental Immunochemistry, 2nd edn, p. 149. Springfield, Illinois: Thomas.

Johnston, K. H., Holmes, K. K. \& GotsChlich, E. C. (1976). The serological classification of Neisseria gonorrhoeae. I. Isolation of the outer membrane complex responsible for serotype specificity. Journal of Experimental Medicine 143, 74I-758.

Penn, C. W., Sen, D., Veale, D. R., Witt, K., Parsons, N. J. \& Smith, H. (1976). Morphological, biological and antigenic properties of Neisseria gonorrhoeae adapted to growth in guinea-pig subcutaneous chambers. Journal of General Microbiology 97, 35-43.

Scales, R. W. \& Kraus, S. J. (I974). Development of passive transfer of immunity to gonococcal infection in guinea pigs. Infection and Immunity 10, 1040-1043.

TURNER, W. H. \& NovotNy, P. (1976). The inability of Neisseria gonorrhoeae pili antibodies to confer immunity in subcutaneous guinea-pig chambers. Journal of General Microbiology 92, 224-228.

Veale, D. R., Sen, D., Penn, C. W., Finch, H. \& Smith, H. (1977). Interaction of Neisseria gonorrhoeae with guinea pig defence mechanisms in subcutaneously implanted chambers. FEMS Microbiology Letters (in the Press).

Veale, D. R., Smith, H., Witt, K. \& Marshall, R. B. (1975). Differential ability of colonial types of Neisseria gonorrhoeae to produce infection and an inflammatory response in subcutaneous perforated plastic chambers in guinea pigs and rabbits. Journal of Medical Microbiology 8, 325-335.

Witt, K., Veale, D. R., Finch, H., Penn, C. W., Sen, D. \& Smith, H. (1976). Resistance of Neisseria gonorrhoeae grown in vivo to ingestion and digestion by phagocytes of human blood. Journal of General Microbiology 96, 34I-350. 